\title{
Belgeo
}

Revue belge de géographie

$4 \mid 2003$

The dynamics of metropolisation : from words to territory

\section{Vandermotten, P. Marissal (avec la collaboration de A. Dubreucq et J.C. Defraigne), La production des espaces économiques, Tome II}

Bruxelles, Editions de l'Université de Bruxelles, 2003

\section{Julien Vandeburie}

\section{OpenEdition}

\section{Journals}

Electronic version

URL: http://journals.openedition.org/belgeo/16752

DOI: 10.4000/belgeo.16752

ISSN: 2294-9135

\section{Publisher:}

National Committee of Geography of Belgium, Société Royale Belge de Géographie

\section{Printed version}

Date of publication: 30 December 2003

ISSN: 1377-2368

\section{Electronic reference}

Julien Vandeburie, « C. Vandermotten, P. Marissal (avec la collaboration de A. Dubreucq et J.C.

Defraigne), La production des espaces économiques, Tome // », Belgeo [Online], 4 | 2003, Online since 14 February 2016, connection on 21 April 2019. URL : http://journals.openedition.org/belgeo/16752 ; DOI : 10.4000/belgeo.16752

This text was automatically generated on 21 April 2019

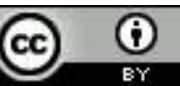

Belgeo est mis à disposition selon les termes de la licence Creative Commons Attribution 4.0 International. 


\title{
C. Vandermotten, P. Marissal (avec la collaboration de A. Dubreucq et J.C. Defraigne), La production des espaces économiques, Tome II
}

Bruxelles, Editions de l'Université de Bruxelles, 2003

\author{
Julien Vandeburie
}

\section{REFERENCES}

C. Vandermotten, P. Marissal (avec la collaboration de A. Dubreucq et J.C. Defraigne) (2003), La production des espaces économiques, Tome II, Bruxelles, Editions de l'Université de Bruxelles

On attendait avec impatience la suite de l'imposant ouvrage de géographie économique des spécialistes de l'ULB. Nous n'avons pas été déçus. Le livre regorge d'informations pertinentes et continue le tour du monde entamé dans le tome précédent, lequel contenait les analyses de la production économique des espaces belges et européens. Le présent tome commence par les Etats-Unis, en expliquant clairement les conditions de son intégration dans le centre de l'économie-monde mais aussi des nombreuses différences internes au pays, les fameuses «ceintures ». Ensuite les auteurs se tournent vers la périphérie et en examinent les conditions socio-économiques. Le monde agricole, l'industrialisation, l'urbanisation l'éducation sont étudiés avant une très bonne conclusion sur les réalités de l'intégration de la périphérie. Le chapitre VII envisage les liens entre croissance démographique et développement par de nombreux calculs et exemples. Le chapitre VIII, consacré à la formation du système de l'économie-monde, est le chapitre central de ce tome, voire des deux tomes qui constituent l'ouvrage. La structuration du centre ayant été examinée dans le premier tome, il s'agit davantage ici 
de la mise en place de la géographie des relations centre-périphérie. Procédant par ordre chronologique, les auteurs étudient par des exemples tels que l'Inde, le pétrole, des multinationales comme celles du textile et de l'automobile, des enjeux d'un intérêt certain. La dernière partie du chapitre, relative aux transactions internationales et à la géographie du commerce mondial, est très instructive. Enfin, le dernier chapitre s'intéresse aux structures spatiales des pays de la périphérie. Les monographies (R.D. Congo, Côte d'Ivoire, Brésil, Chili, Mexique, Algérie, Iran, Corée du sud, Chine) sont très bien faites, quoiqu'on puisse trouver un peu courts les commentaires sur la Chine. Un exemple d'ancienne économie planifiée aurait été bienvenu. En outre, on se demande parfois à quoi servent toutes ces cartes et tous ces tableaux. Ils apparaissent sous-utilisés dans de nombreux cas, par exemple les figures 135, 202, 213. La qualité des cartes laisse parfois à désirer : dans les figures 158,160,161, 183 et 212 par exemple, on ne peut pas distinguer les deux dernières classes. En conclusion l'ouvrage passionnera les connaisseurs mais impressionnera peut-être les néophytes, son contenu dense restant une de ses marques de fabrique. 